\title{
Diminuindo a desigualdade no acesso à Internet entre estudantes alfabetizados até a entrada na Universidade
}

\author{
Juliao Braga $^{1}$, Ana Leda Silva Moraes ${ }^{2}$, Jéferson Nobre ${ }^{3}$, Marcelo Santos ${ }^{4}$ \\ ${ }^{1}$ Universidade Presbiteriana Mackenzie (UPM) \\ ${ }^{2}$ Universidade Federal do Estado de São Paulo (UNIFESP) \\ ${ }^{3}$ Universidade Federal do Rio Grande do Sul (UFRGS) \\ ${ }^{4}$ Instituto Federal do Sertão Pernambucanno \\ j@braga.net.br, analedamoraes@gmail.com, \\ jcnobre@inf.ufrgs.br, marcelo.santos@ifsertao-pe.edu.br
}

\begin{abstract}
This work describes a project that aims to reduce the inequality of opportunities in Internet access in Brazil and can be used to meet a similar objective in other countries. Basic education students, living in peripheral communities or in remote locations and participating in rural public schools, among others, have difficulties in accessing the Internet. With this they stop participating in the largest repository of knowledge in the world, missing great opportunities for personal growth in the serene construction of a future of their choice.
\end{abstract}

Resumo. Este trabalho descreve um projeto que visa diminuir a desigualdade de oportunidades no acesso à Internet no Brasil podendo ser aproveitado para atender objetivo semelhante em outros países. Estudantes do ensino básico, residentes em comunidades periféricas ou em localidades remotas e participantes de escolas públicas rurais, entre outras, têm dificuldades no acesso à Internet. Com isto deixam de participar do maior repositório de conhecimento do mundo, perdendo grandes oportunidades de crescimento pessoal na construção serena de um futuro de sua escolha.

\section{Introdução}

Este trabalho descreve um projeto que visa diminuir a desigualdade de oportunidades no acesso à Internet no Brasil, podendo ser aproveitado, inclusive, em outros países com objetivo semelhante.

Estudantes do ensino fundamental, médio, médio-técnico e participantes da educação de jovens e adultos residentes em comunidades periféricas ou em localidades remotas e participantes de escolas públicas rurais, entre outras, têm dificuldades no acesso à Internet. Com isto deixam de participar do maior repositório de conhecimento do mundo, perdendo grandes oportunidades de crescimento pessoal na construção serena de um futuro de sua escolha.

O denominado Projeto Iter (ITER) diminui esta desigualdade por meio de:

- Baixo custo de implementação

- Capilaridade 
- Baixo custo operacional

- Rapidez na implementação

- Segurança

produzindo ações que tornem efetivas:

- a disponibilidade de tráfego e acesso à Internet aos estudantes, desde a alfabetização até a entrada na Universidade;

- disponibilizar e manter tráfego de Internet nas escolas públicas secundárias;

- disponibilizar e manter tráfego de Internet em ambientes comunitários e nas moradias dos estudantes;

- o apoio a redes de Internet comunitárias sem o envolvimento direto do ITER, com as respectivas atividades operacionais.

O ITER propõe atender aos objetivos delineados acima, em duas ações paralelas:

1. Facilitar o uso da Internet através dos mais de 2.600 pontos do eduroam no Brasil;

2. Utilizar-se da imensa capilaridade de acesso dos pequenos, médios e grandes Provedores de Serviços de Internet (ISPs), devidamente remunerados por esta iniciativa.

Para se assegurar da viabilidade destes objetivos, o ITER passará por uma implementação de teste durante o período de quinze (15) meses, em todo o Brasil. A esta implementação se dará o nome de Prova de Conceito (PC). Isto acontecerá através de um esforço conjunto de Colaboradores e Parceiros (pessoas físicas e jurídicas) registrando as lições aprendidas durante a PC e exibindo seus resultados nos mínimos detalhes. Destas experiências e anotações o ITER será aperfeiçoado. O repositório das informações sobre o ITER está disponível no ambiente do Open Science Framework ${ }^{1}$ (OSF).

Este trabalho está dividido em nove seções, incluindo a Introdução. A Seção 2 é uma apresentação sumária do eduroam. A Seção 3 apresenta a topologia do ITER e sua relação com o eduroam e os Provedores de Acesso à Internet (ISPs), abordando o RADIUS como uma solução para a proposta da arquitetura do ITER descrita na Seção 4. A Seção 5 apresenta as estratégias operacionais do ITER, sua governança e a participação das múltiplas partes interessadas, caracterizando o apoio institucional da ISOC Brasil (capítulo brasileiro da Internet Society). A Seção 6 apresenta as etapas iniciais para implementar o projeto e estabelecer os primeiros documentos de governança. A seção 7 apresenta um resumo dos trabalhos relacionados. A Seção 8 apresenta o cronograma da implementação do período de teste do ITER. A Seção 9 apresenta um sumário das conclusões e trabalhos futuros associados ao projeto.

\section{O eduroam}

O eduroam é uma iniciativa criada em 2003 e hoje está funcionando em mais de 100 países, com orientação fortemente acadêmica [Wierenga et al. 2015]. Sua abrangência pode ser vista na Figura 1.

Ele é estabelecido por uma tecnologia baseada no padrão IEEE 802.1X e em um conjunto de servidores baseados no protocolo RADIUS (um acrônimo de Remote Access

\footnotetext{
${ }^{1}$ https://osf.io/mrkvw/
} 


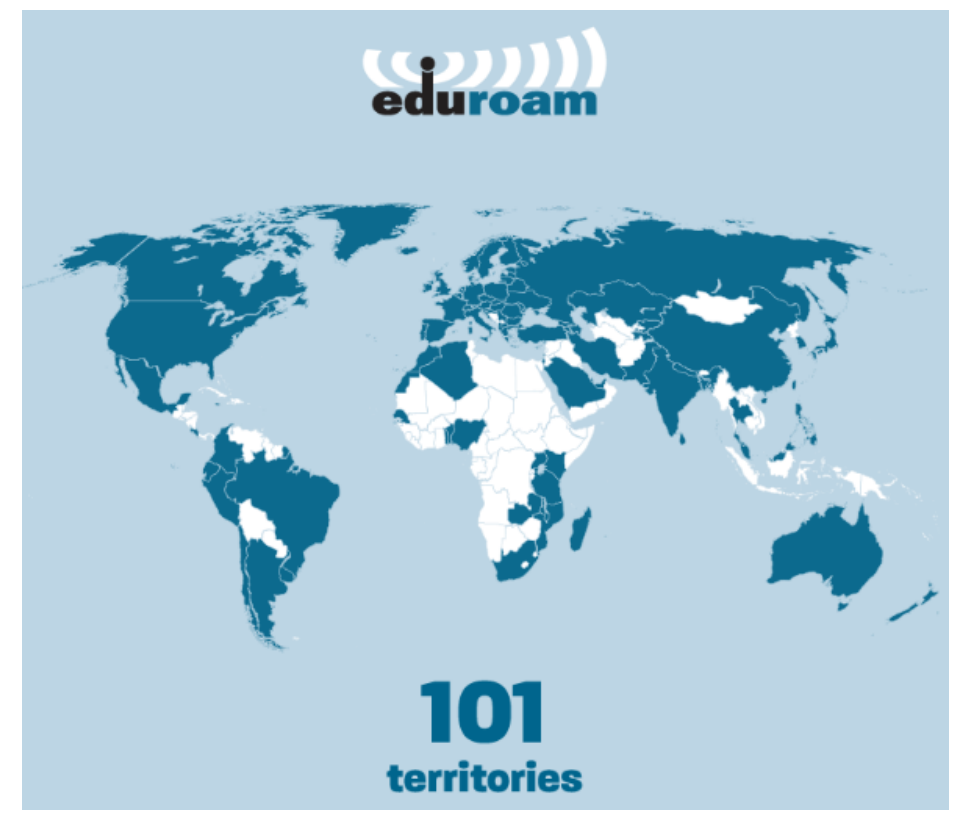

Figure 1. Alcance do eduroam. Fonte: https://www.eduroam.org/ wp-content/uploads/eduroam-growing-topology-v2.gif

Dial In User Service), definido pelo Internet Engineering Task Force (IETF) em 1997, descrito e atualizado pela RFC2865 [Rigney et al. 2000].

Técnicas disponíveis no RADIUS possibilitam facilidades que o eduroam explora e percebíveis em seu funcionamento, como mostra a Figura 2.

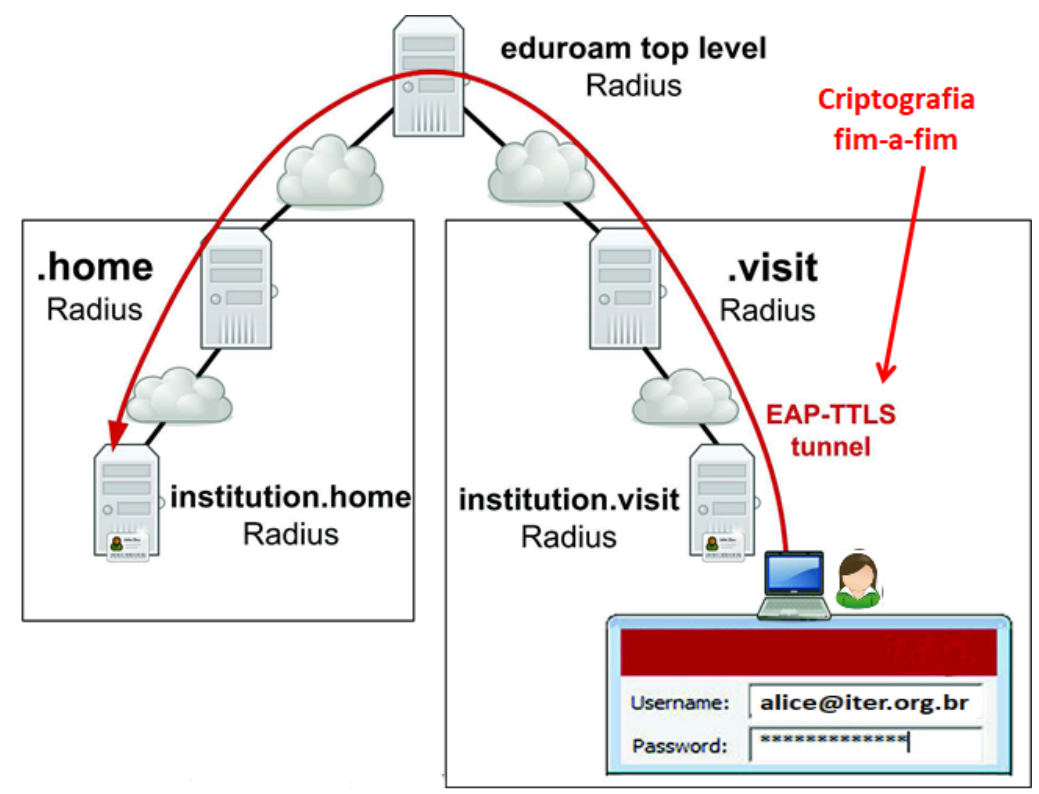

Figure 2. 0 funcionamento do eduroam. Adaptado de: https ://www . eduroam . org/how

Como exemplo, Alice é uma estudante com uma identificação (ID) do ITER, isto é, ela possui um nome associado ao domínio do projeto e uma senha, que representa- 
mos como uma dupla: [alice@iter.org.br, senha]. Ela estava visitando a biblioteca de uma Universidade, no interior de Pernambuco e percebeu que havia eduroam por lá. Na figura acima, é a institution.visit. Alice captou o sinal no seu celular e se conectou ao RADIUS da institution. visit, como mostra a figura. O RADIUS não reconheceu alice@iter.org.br e encaminhou uma mensagem criptografada para um servidor RADIUS da federação brasileira do eduroam, perguntando se conhecia alice@iter.org.br. O RADIUS da federação reconheceu que a instituição do domínio iter.org.br era o domínio do ITER e enviou os dados da solicitação para o RADIUS do ITER que é a institution.home da Alice. Este, por sua vez, informa diretamente e criptografado, ao RADIUS da institution.visit, que Alice é conhecida e, complementarmente, sua senha correta. Assim, Alice navegou na Internet! Este processo durou alguns poucos segundos...

O eduroam no Brasil é gerenciado pela Rede Nacional de Ensino e Pesquisa ${ }^{2}$ (RNP) com mais de 2.600 pontos de acesso disponíveis.

\section{O modelo do ITER}

O eduroam se aproveita das técnicas disponíveis no RADIUS [Hassell 2002] ou, mais efetivamente, disponíveis no freeRadius ${ }^{3}$ [Van der Walt 2011]. O ITER se apropria das facilidades propiciadas pelo eduroam e, também, pelas técnicas disponibilizadas pelo freeRadius. A explicação do ITER segue a exibição de seu modelo, na Figura 3.

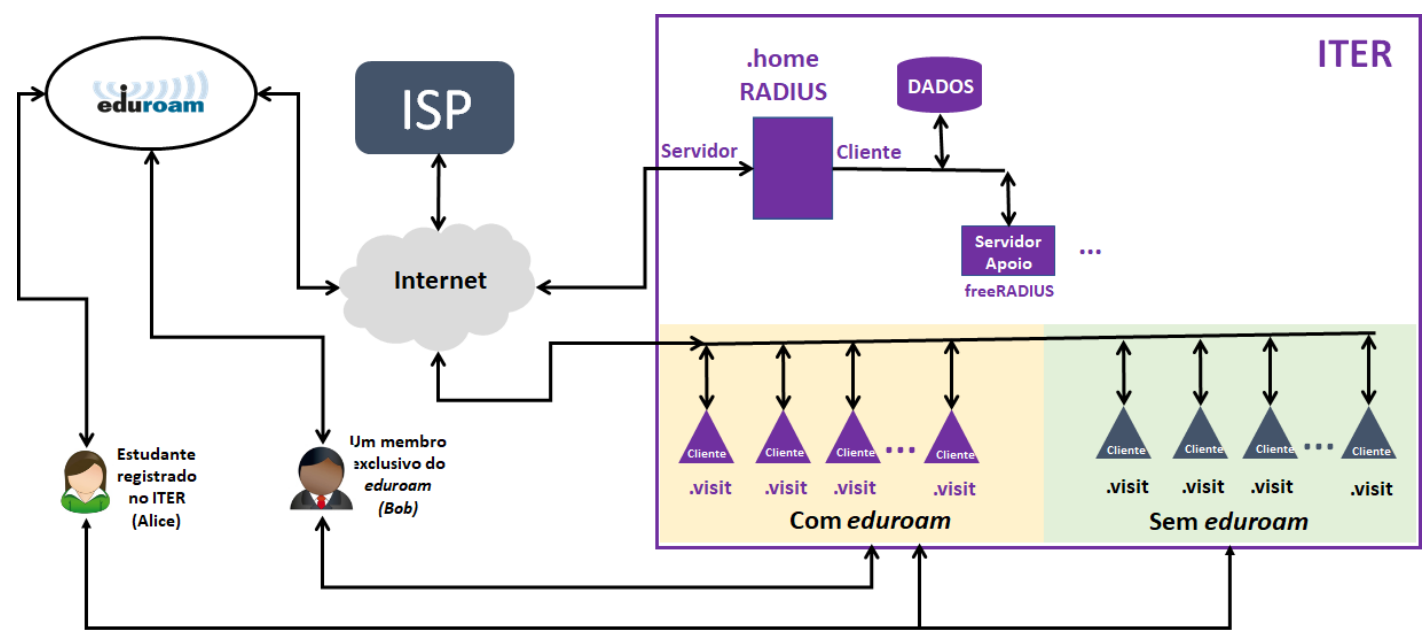

Figure 3. O Modelo do Projeto ITER.

No retângulo em roxo à direita, estão os recursos do ITER e a relação com seus usuários e facilidades (como o eduroam), à esquerda. Além da Alice, estudante registrada no ITER, um outro personagem aparece para entendermos o funcionamento do ITER: trata-se de Bob, um personagem registrado apenas no eduroam.

Alice, por estar registrada no ITER tem a facilidade de usar a Internet tanto nos ambientes do ITER, que são associados ao eduroam, quanto naqueles que não estão no eduroam, mas que estão disponíveis pelos ISPs parceiros do ITER. Bob só consegue acessar a Internet por um . visit do eduroam, vários deles disponíveis pelo ITER. Esta diferença

\footnotetext{
${ }^{2} \mathrm{https}: / /$ www.rnp.br/servicos/alunos-e-professores/colaboracao-a-distancia/eduroam

${ }^{3}$ https://freeradius.org/
} 
na facilidade de acesso da Alice em relação ao Bob se deve ao fato que o ITER faz acordo com os provedores para usar a imensa capilaridade de difusão do sinal da Internet que eles possuem. Os membros do eduroam se entendem entre si, porque possuem servidores RADIUS, os quais permitem uma forte colaboração e interconectividade entre eles. $\mathrm{O}$ ITER também possui servidores RADIUS na sua infraestrutura preparados para, oportunamente, se interconectarem com os servidores RADIUS da federação do eduroam, no Brasil disponíveis pela RNP.

Estes mesmos servidores RADIUS do ITER são conectados com os servidores RADIUS dos ISPs parceiros que disponibilizam o acesso aos estudantes, como a Alice, através de seus inúmeros pontos de acessos capilarizados com o protocolo IEEE 802.1X.

\section{A Estrutura da Educação Básica Brasileira}

A Lei no. 9.384 chamada de Lei das Diretrizes e Bases da Educação Nacional estabelece em seu Artigo 21: A educação escolar compõe-se de: I - educação básica, formada pela educação infantil, ensino fundamental e ensino médio; II - educação superior [Presidencia da Republica, Brasil 1996]. De forma gráfica isto está representado na Figura 4.

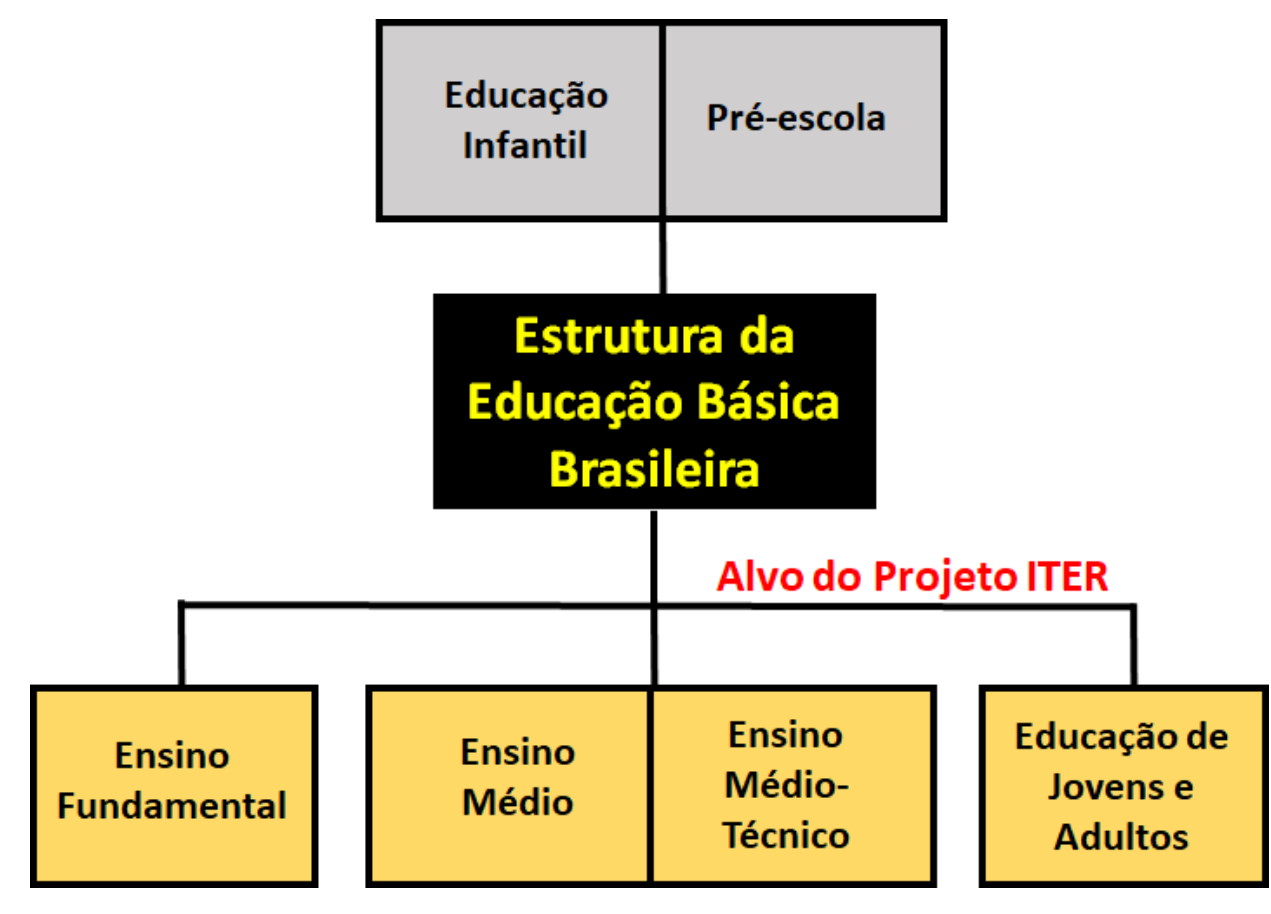

Figure 4. Organização do ensino básico brasileiro. O Alvo do Projeto ITER está representado nos retângulos em amarelo

O Instituto Nacional de Estudos e Pesquisas Educacionais Anísio Teixeira (INEP) organiza o censo educacional brasileiro. O censo de 2019 contém os dados que permitiram identificar aqueles dados - do ensino fundamental e médio - com os quais o Projeto ITER estará envolvido em sua fase de testes [INEP-MEC 2019]. Estes dados divididos pelas respectivas unidades da federação estão re-presentados na Tabela 1, indicando que aproximadamente 39 milhões de estudantes podem se habilitar ao uso do ITER distribuídos por quase 154 mil escolas públicas (federais, estaduais e municipais) e privadas por todo o país. 
Table 1. Matriculas e escolas em 2019

\begin{tabular}{|r|l|r|r|}
\hline$\#$ & \multicolumn{1}{|c|}{ Estados } & \multicolumn{1}{c|}{ Alunos } & Escolas \\
\hline 1 & Acre & 220.422 & 2.099 \\
2 & Alagoas & 730.819 & 2.857 \\
3 & Amapá & 192.199 & 856 \\
4 & Amazonas & 910.793 & 5.14 \\
5 & Bahia & 2.944 .008 & 15.023 \\
6 & Ceará & 1.767 .618 & 6.373 \\
7 & Distrito Federal & 548.911 & 1.105 \\
8 & Espírito Santo & 713.389 & 2.501 \\
9 & Goiás & 1.192 .534 & 4.365 \\
10 & Maranhão & 1.627 .092 & 10.496 \\
11 & Mato Grosso & 705.055 & 2.621 \\
12 & Mato Grosso do Sul & 542.745 & 1.554 \\
13 & Minas Gerais & 3.531 .194 & 13.751 \\
14 & Paraná & 2.056 .934 & 8.195 \\
15 & Paraíba & 792.227 & 4.064 \\
16 & Pará & 1.968 .917 & 10.232 \\
17 & Pernambuco & 1.822 .776 & 8.040 \\
18 & Piauí & 751.436 & 3.950 \\
19 & Rio Grande do Norte & 563.130 & 3.067 \\
20 & Rio Grande do Sul & 1.815 .671 & 6.348 \\
21 & Rio de Janeiro & 2.894 .081 & 9.996 \\
22 & Rondônia & 357.025 & 1.024 \\
23 & Roraima & 159.211 & 811 \\
24 & Santa Catarina & 1.211 .680 & 4.103 \\
25 & Sergipe & 455.216 & 2.051 \\
26 & São Paulo & 7.655 .052 & 21.649 \\
27 & Tocantins & 329.774 & 1.613 \\
\hline & \multicolumn{1}{|c|}{ Total } & $\mathbf{3 8 . 4 5 9 . 9 0 9}$ & $\mathbf{1 5 3 . 8 8 4}$ \\
\hline
\end{tabular}

\section{Arquitetura do Projeto ITER}

O ecossistema do ITER é bastante parecido com o eduroam [Wierenga et al. 2015], com algumas características operacionais especiais descritas abaixo e que podem ser vistas na Figura 5. Na realidade, a arquitetura do ITER usa o que há de melhor na estrutura do eduroam e nas facilidades disponíveis pelo RADIUS.

- Ele atende a dois ambientes: o ambiente do eduroam e o ambiente específico do próprio ITER. Os servidores RADIUS de ambos os ambientes são logicamente apresentados como uma hierarquia semelhante ao esquema de resolução de nomes pelo servidor de DNS, onde em cada ambiente existe um RADIUS raiz. No caso do eduroam, cada país possui um RADIUS raiz.

- O seu RADIUS do topo da hierarquia (servidor remoto) atua somente como Provedor de Identidade (IdP), isto é, reconhece um estudante previamente cadastrado no ITER.

- Todos os outros RADIUS, tanto do ambiente eduroam, como do ambiente dos ISPs atuam como Provedores de Serviços (SPs). 


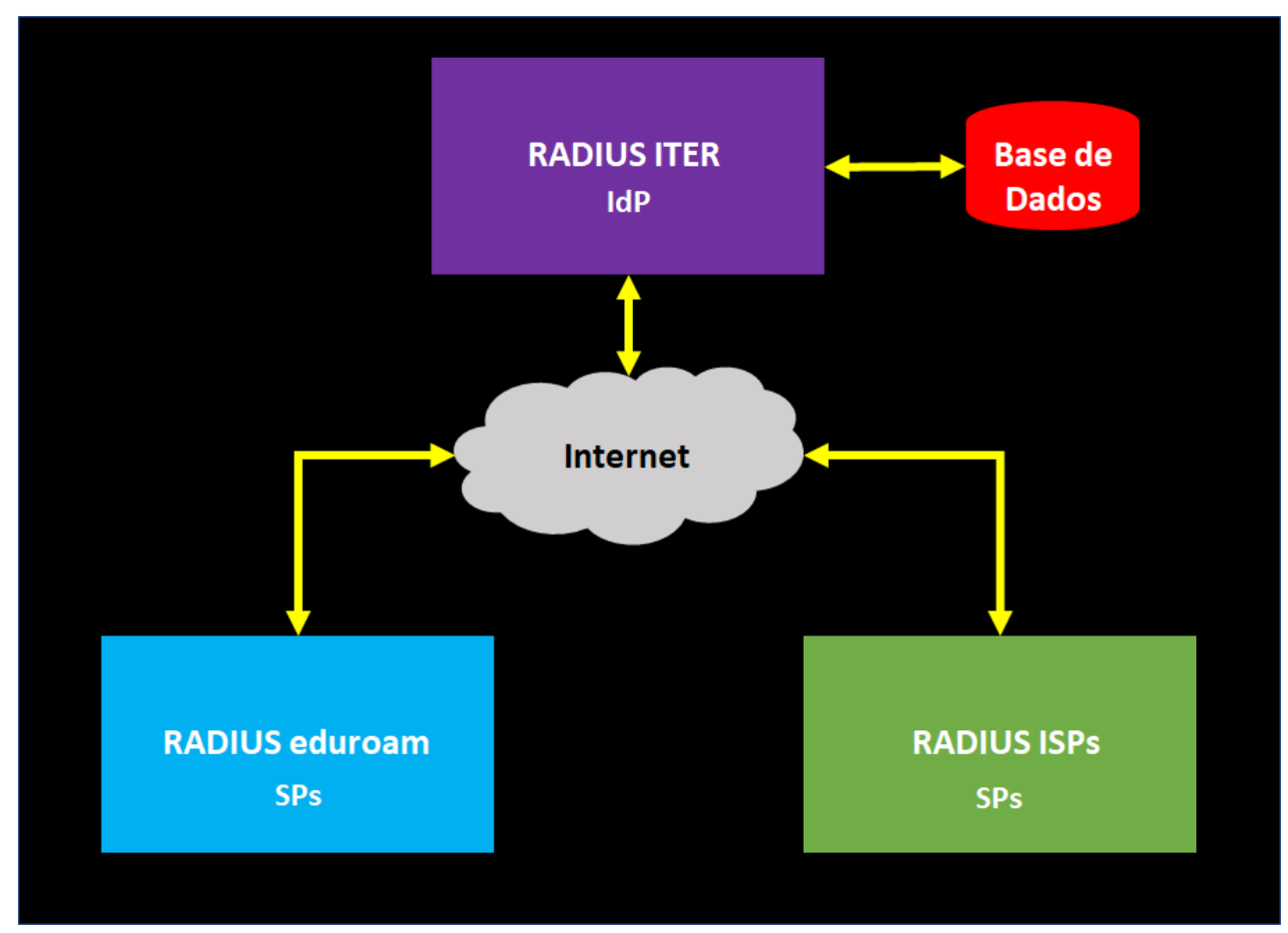

Figure 5. Arquitetura do ITER: atua somente como Provedor de Identidade (IdP)

- O ambiente do eduroam reconhece o RADIUS do ITER através de seu servidor RADIUS no domínio da federação brasileira, sob a administração da RNP.

- Os servidores RADIUS do ambiente do próprio ITER estão administrados pelos ISPs aderentes ao Projeto ITER. Eles são SPs e atuam como proxies, na concepção do RADIUS [Rigney et al. 2000]: o servidor RADIUS de um ISP recebe uma requisição de autenticação de um RADIUS (por exemplo um Network Access Server (NAS)) e encaminha diretamente para o RADIUS do ITER, conectando através de seu domínio [Wierenga et al. 2015].

- O mecanismo de autenticação é uma combinação do IEEE 802.1X [IEEE 2010] e do Extensible Authentication Protocol (EAP) [Adrangi et al. 2006, Aboba et al. 2004, Alvestrand 2004, Stanley et al. 2005]. Este é o processo de autenticação do eduroam e adotado, também, pelo ambiente do ITER.

- Este mecanismo de autenticação levado a cabo por um servidor RADIUS, atuando como um proxy, recebe a denominação de suplicante e age igualmente em qualquer um dos ambientes usando a estrutura de confiança ${ }^{4}$ do eduroam, adotando o formato do Network Access Identifier (NAI) (por exemplo, alice@iter.org.br) [Winter and McCauley 2015, DeKok 2015], usado pelo RADIUS. A figura 6 ilustra este comportamento, associando-o ao EAP e ao protocolo TLS, inerentes ao RADIUS [Dierks and Rescorla 2008, Winter et al. 2012, Eronen and Tschofenig 2005] com as facilidades do Tunnel Extensible Authentication Protocol (TEAP), um método de tunelamento que permite comunicação segura entre um cliente e um servidor, usando o TLS para estabelecer um túnel mutuamente autenticado [Zhou et al. 2014].

- Toda a atenção será dada à questão de segurança. Assim, o hospedeiro da estru-

\footnotetext{
${ }^{4}$ Tradução livre do inglês trust fabric
} 


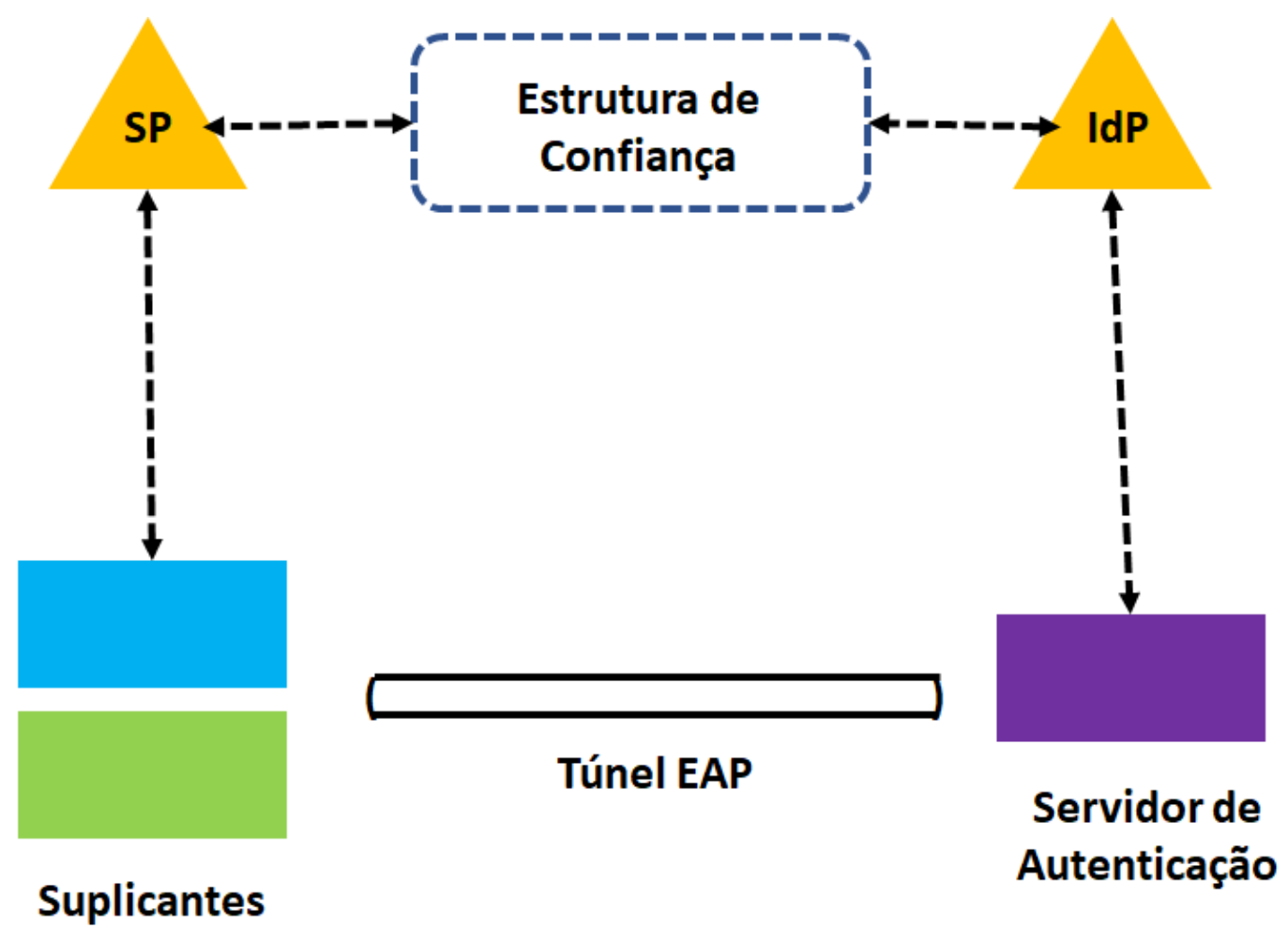

Figure 6. Tunelamento EAP. Adaptado da RFC7593 [Wierenga et al. 2015]

tura do ITER terá de se assegurar que segue as recomendações e participação no Mutually Agreed Norms for Routing Security ${ }^{5}$ (MANRS).

\section{Estratégias operacionais do projeto}

Para viabilizar o ITER está sendo proposto um conjunto de estratégias que culminam na mais importante, que é a Prova de Conceito (PC).

As estratégias operacionais propostas: governança, remuneração dos ISPs, uso parcial do eduroam e PC, descritas na sequência.

\subsection{Governança}

O projeto ITER está institucionalmente vinculado à ISOC Brasil, que exerce papéis de aconselhamento e supervisão, assim como de apoio no relacionamento institucional com outras entidades e na busca de patrocínios. Mas a governança do projeto é feita por sua própria equipe, que se relaciona com as múltiplas partes interessadas denominadas, genericamente, de entidades, com o objetivo de torná-lo operacional. A organização da estrutura de governança do ITER está em elaboração e a Figura 7 exibe a relação funcional entre a ISOC Brasil, o comitê de coordenação do projeto e as múltiplas partes interessadas.

As partes interessadas são os voluntários, os colaboradores, os parceiros e as organizações patrocinadoras. Todas elas se juntam para garantir que o objetivo do ITER se concretize: atender aos alunos do ensino fundamental e médio, a principal parte interessada e denominado alvo. Agrupadas de forma organizada pelo comitê de coordenação do

${ }^{5}$ https: / / www . manrs.org/ 


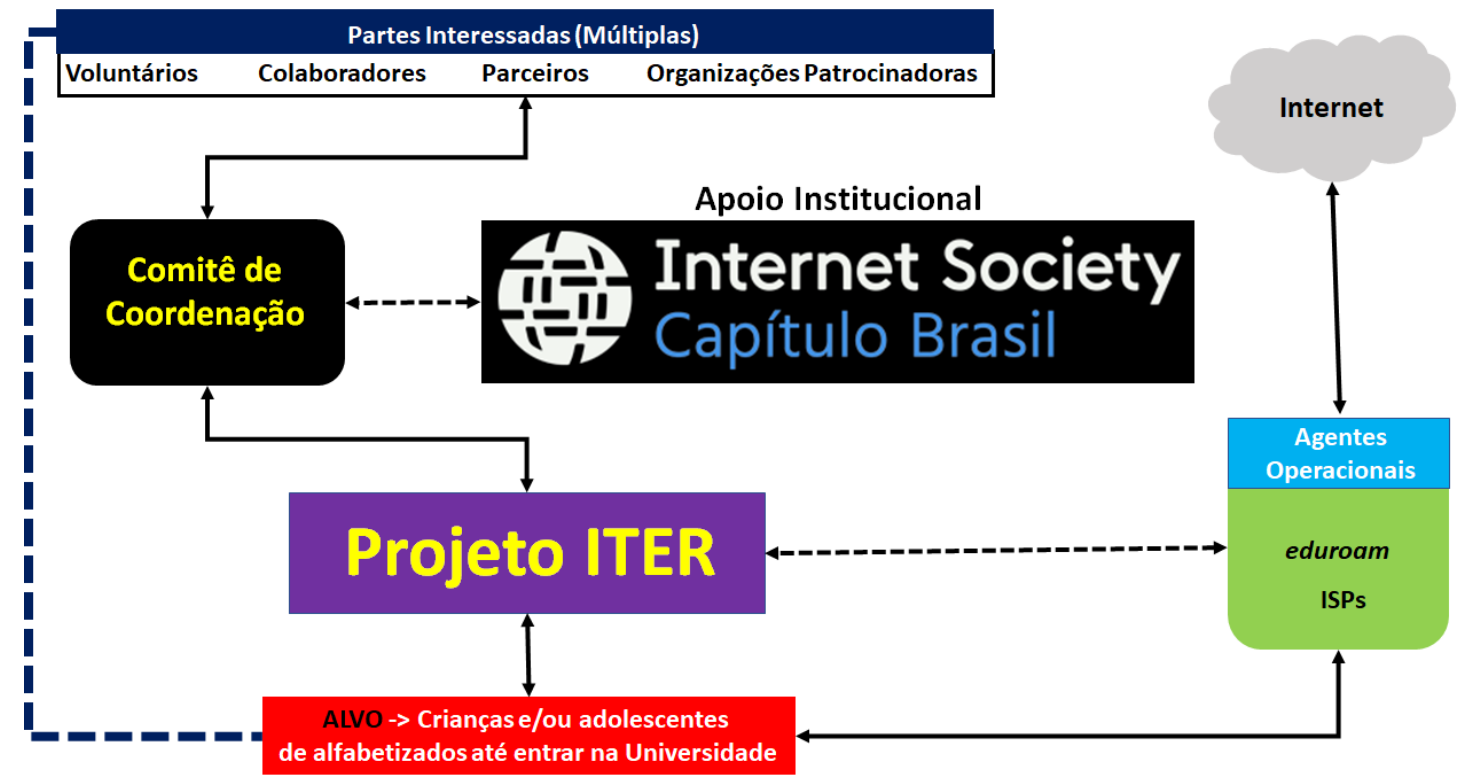

Figure 7. A governança do Projeto ITER. com seu Comitê de Coordenação, o apoio institucional da ISOC Brasil e as múltiplas partes interessadas

projeto, sob o abrigo institucional da ISOC Brasil, as entidades interferem na organização do ITER através de um processo que permite recomendações para alteração dos estatutos, à semelhança da participação da sociedade nas políticas da Internet para a América Latina e Caribe, promovida pelo Registro de Endereçamento da Internet a América Latina e o Caribe $^{6}$ (LACNIC).

Os voluntários são as entidades (pessoas, instituições, organizações, etc.) que disponibilizam trabalho parcial ou total ao projeto, sem remuneração. Fazem isto permanentemente ou temporariamente. O aceite e avaliação dos voluntários para o ITER é responsabilidade do Comitê de Coordenação do projeto.

Os colaboradores são as entidades (pessoas, instituições, organizações, etc.) que exercem suas habilidades em demandas do ITER e são remuneradas por seu trabalho. A administração dos voluntários é responsabilidade do comitê de coordenação do projeto.

Os parceiros são os principais interessados no projeto sob o ponto de vista operacional. Incluem as escolas (públicas e privadas) do ensino básico até o ensino profissional e, também, os ISPs. Cumpre ao Comitê de coordenação do projeto a convocação, cadastramento e orientação e, quando for o caso remunerar os parceiros.

As organizações patrocinadoras são as responsáveis pelo financiamento do ITER, isto é, da remuneração dos parceiros, investimentos e custos operacionais projetados pelo comitê de coordenação do projeto.

Entidades podem participar de uma ou mais partes interessadas e se relacionam entre si, como na Figura 8, tornando-se não caracterizadas na intersecção de todas elas.

\footnotetext{
${ }^{6}$ https://www.lacnic.net/1042/3/lacnic/acerca-do-lacnic
} 


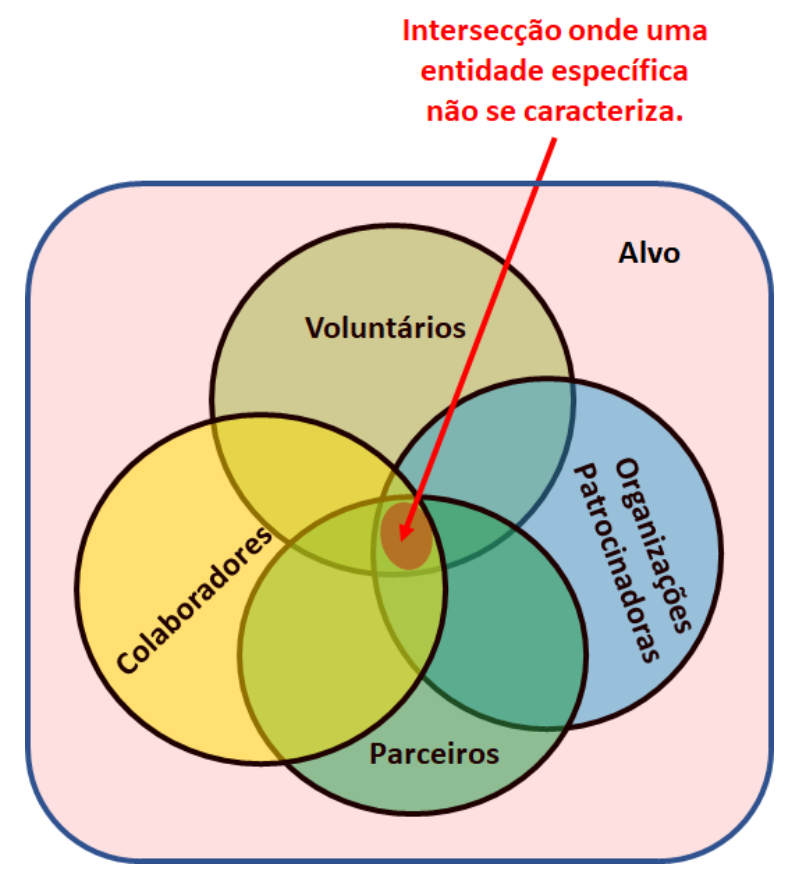

Figure 8. Uma entidade pode participar caracterizada como diversas partes interessadas. Entretanto, na intersecção ela não se caracteriza.

\subsection{Remuneração dos ISPs}

No mês de março de 2020, a Agência Nacional de Comunicações (Anatel) divulgou que os ISPs de pequeno porte estão se colocando em uma posição confortável entre os grandes operadores do setor. Esta evolução, ao longo dos anos, pode ser vista na Figura 9.

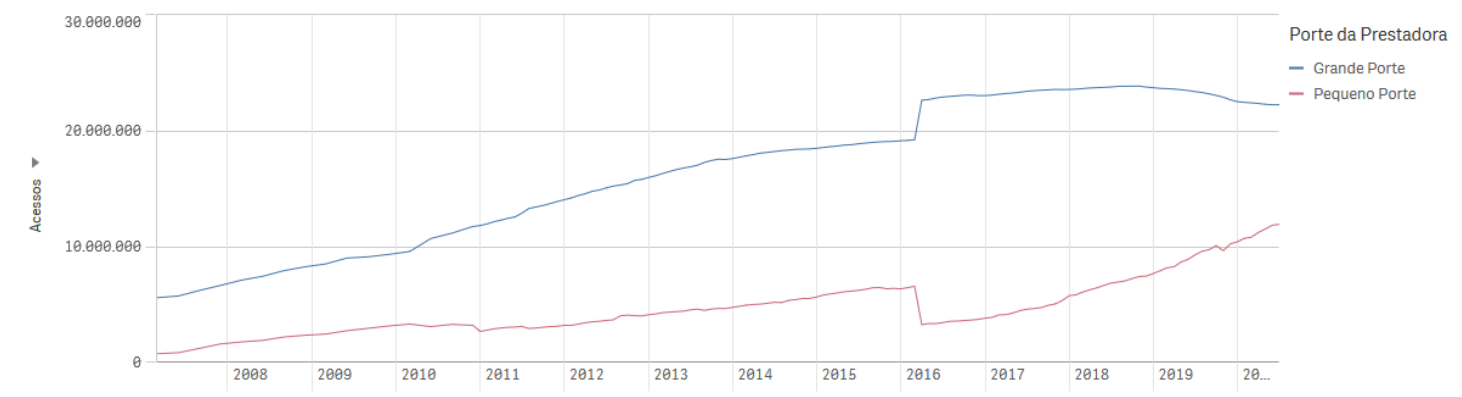

Figure 9. Evolução dos acessos de banda larga fixa por porte da prestadora e empresa. As 10.405 entidades cadastradas na Anatel produziram um total de 34.181 .324 acessos $(65,10 \%)$, provedores de grande porte respondem por 22.249.889 acessos e provedores de pequeno porte respondem por 11.931 .455 acessos (34,90\%), em julho de 2020, Fonte: https://www.anatel.gov.br/ paineis/acessos/banda-larga-fixa

Com isto eles estão, com o passar do tempo aumentando a capilaridade e disponibilizando o acesso à Internet também, nos locais mais remotos do território brasileiro.

Para o ITER eles são os parceiros ideais e complementares aos dos grandes provedores.

Como forma de expandir a capilaridade com maior rapidez e atingindo locais es- 
tratégicos, sob o ponto de vista do atendimento do alvo, eles precisam e devem ser remunerados de forma adequada. O Comitê de Coordenação, onde representantes dos ISPs estarão presentes estabelecerá a remuneração adequada e justa aos ISPs após a execução da PC.

\subsection{O uso do eduroam}

O eduroam é uma oportunidade de tornar disponível para o alvo o imediato acesso à Internet através de seus mais de 2.600 pontos espalhados pelo Brasil. Depende somente de entendimentos com a RNP, quem deverá aprovar a sua utilização no Brasil.

\subsection{Prova de Conceito}

Alguns voluntários estão desenvolvendo protocolos que estabeleçam uma cama de testes à qual está se denominando prova de conceito $(\mathrm{PC})$.

A PC para o ITER é o funcionamento por um período de um ano, do modelo integral proposto na Figura 3. As etapas que envolvem a preparação para a execução da PC são as seguintes:

1. Convocação dos ISPs interessados e que possuam RADIUS em sua infraestrutura e que estejam dispostos a participar neste primeiro ano, da prova de conceito, sem remuneração;

2. Convocação de escolas de primeiro e segundo graus, particularmente de locais remotos mas que possuam cobertura de Internet através de um ISP;

3. Convocação de colaboradores voluntários para participação dos debates que estabelecerão as definições técnicas;

4. Instituições não governamentais que possam orientar a equipe de voluntários a estabelecer as políticas de aproximação da periferia;

5. Convocação de fabricantes e/ou representantes de fabricantes de equipamentos aderentes ao projeto ITER, como por exemplo os Network Access Server (NAS);

6. Organizações Patrocinadoras interessadas em participar das regras que irão delinear o futuro do ITER e de que maneira seriam feitos os aportes financeiros para manter o tráfego via ISPs, após o PC.

\section{Trabalhos Relacionados}

Antes da Internet se tornar comercial, em s1993, já havia preocupações em drelação à desigualdade digital, incluindo esforço para caracterizá-la [Van Dijk 2006]. Países da América Latina, como o Uruguai propuseram soluções envolvendo redes comunitárias, ideia que se espalhou em várias regiões do Brasil [Rivoir Cabrera 2009]. Sociólogos se debruçaram para identificar e propor soluções para a inclusão social e as tecnologias de comunicação. Estas questões, embora muito importantes fogem do escopo do presente trabalho, Por outro lado, o que se conseguiu verificar em pesquisa bibliográfica é de que o modelo proposto para o ITER, particularmente no que diz respeito a participação dos ISPs segue uma alternativa original.

\section{Cronograma das atividades iniciais}

A Tabela 2 identifica os principais componentes necessários à alavancagem do ITER e de sua PC. Como qualquer projeto, estes componentes sofrem alterações ao longo do tempo necessitando, portanto, de uma coordenação permanente do projeto. 
Table 2. Cronograma preliminar das principais etapas que envolvem o ITER

\begin{tabular}{|c|c|c|c|c|}
\hline \# & Atividade & Responsabilidade & Início & Fim \\
\hline 1 & Projeto técnico da $\mathbf{P C}$ & $\mathrm{JB}^{a}, \mathrm{LL}^{b}, \mathrm{MS}^{c}, \mathrm{JN}^{d}$ & set $/ 20$ & out $/ 20$ \\
\hline 2 & Operação da PC & $\mathrm{JB}^{a}, \mathrm{LL}^{b}, \mathrm{MS}^{c}, \mathrm{JN}^{d}$ & nov/20 & Out/21 \\
\hline 3 & Operação do Projeto ITER & Comitê de Coordenação & out/21 & - \\
\hline 4 & Plano de Marketing & Comitê de Coordenação & set/20 & - \\
\hline 5 & Identificação Única (Garantia) & Comitê de Coordenação & set $/ 20$ & out $/ 20$ \\
\hline 6 & Contrato Parceria ISPs período da PC & Comitê de Coordenação & set/20 & set/20 \\
\hline 7 & Chamada Participação Parceiros ISP & Comitê de Coordenação & out $/ 20$ & - \\
\hline 8 & Chamada Participação Voluntários & Comitê de Coordenação & set/20 & - \\
\hline 9 & Documento de políticas do ITER & Grupo de voluntários & set $/ 20$ & out $/ 20$ \\
\hline 10 & Regras de alteração documento de políticas & Grupo de voluntários & set $/ 20$ & out $/ 20$ \\
\hline 11 & Estrutura da Governança do ITER & Comitê de Coordenação & set $/ 20$ & - \\
\hline 12 & Site do Projeto ITER & Comitê de Coordenação & set $/ 20$ & - \\
\hline 13 & Ações sobre Organizações Patrocinadoras & Comitê de Coordenação & set/20 & - \\
\hline
\end{tabular}

Legenda:

${ }^{a} \rightarrow$ Julião Braga

$b^{b}>$ Luciano I. Lima

${ }^{c} \rightarrow$ Marcelo Santos

$d_{\rightarrow} \rightarrow$ Jeferson Nobre

\section{Conclusões e Trabalhos Futuros}

As Organizações Patrocinadoras somente se aventurarão em apoiar o ITER, se o PC demonstrar a efetividade dos resultados. Isto indica a necessidade de uma atenção especial durante a etapa dos testes que deverá possuir mecanismos para avaliar mudanças de rumos diante de eventuais dificuldades nas ações atuais.

É de se esperar que um dos grandes beneficiários da diminuição da desigualdade escolar, a médio e longo prazos, serão as instituições de ensino superior, em especial as universidades privadas. Portanto, são candidatas as partes interessadas como Organizações Patrocinadoras.

Uma das preocupações que fará parte da etapa da $\mathbf{P C}$ é a eficiência do(s) servidor(es) Radius disponíveis para autenticação, pois a base de dados usará SQL, um mecanismo razoavelmente lento, principalmente na escrita de dados. Adicionalmente, há outros fatores que afetam a eficiência de servidores Radius, que incluem: disponibilidade de memória principal, velocidade da CPU, o mecanismo EAP-TTLS e a complexidade das políticas implementadas ${ }^{7}$. Uma equipe estará disponível com o objetivo de simular e medir a eficiência de configurações que deverão ser introduzidas no ambiente do ITER em sua atividade operacional normal, a qual poderá atender 39 milhões de estudantes (Tabela 1), sob o protocolo AAAA ${ }^{8}$. O Radius (e o freeRadius) é dotado de recursos de simulação de eficiência em diversos cenários que podem ser desenhadas orientados ao ITER, na própria ferramenta radclient e em outras desenvolvidas por terceiros, como a RadPerf $^{9}$. Por outro lado, um servidor Radius pode se comportar como cliente de outro

\footnotetext{
${ }^{7}$ https://wiki.freeradius.org/features/Fast

${ }^{8}$ Implementa os serviços de autenticação (authentication), autorização (authorization), contabilidade (accounting) e auditoria (auditing)

${ }^{9}$ https://networkradius.com/radius-performance-testing/
} 
servidor Radius, o que assegura a escalabilidade modular.

Outros tipos de parceiros irão existir e técnicas para participação destes novos parceiros serão necessárias. Por exemplo, escolas públicas e privadas com enlace de Internet com banda navegável podem participar do eduroam, desde que isto seja permitido pela sua governança no Brasil ou diretamente no ITER. Tais parceiros, usam a Internet como meio e, portanto, não exigirão custos operacionais do ITER mas sim, apoio técnico.

\section{Agradecimentos}

Os autores agradecem:

- ao Professor Dr. Flavio Rech Wagner (ISOC Brasil, UFRGS), pelas sugestões que esclarecem o apoio da ISOC Brasil ao Projeto ITER;

- ao Professor Dr. Demi Getschko (ISOC Brasil, PUC-SP), pelo apoio ao projeto, recomendações e orientações baseadas em sua incomparável experiência;

- ao Engenheiro Luciano Inácio Gonçalves Lima, Diretor Técnico da Jupiter Telecomunicações pela disponibilidade dos recursos materiais necessários à execução da PC.

\section{Referências}

[Aboba et al. 2004] Aboba, B., Blunk, L., Vollbrecht, J., Carlson, J., and Levkowetz, H. (June 2004). Extensible Authentication Protocol (EAP). Technical report, RFC Editor. DOI: $10.17487 /$ RFC3932.

[Adrangi et al. 2006] Adrangi, F., Lior, A., Korhonen, J., and Loughney, J. (January 2006). Chargeable User Identity. Technical report, RFC Editor. DOI: 10.17487/RFC4372.

[Alvestrand 2004] Alvestrand, H. (October 2004). The IESG and RFC Editor Documents: Procedures. Technical report, RFC Editor. DOI: 10.17487/RFC3748.

[DeKok 2015] DeKok, A. (May 2015). The Network Access Identifier. Technical report, RFC Editor. DOI: 10.17487/RFC7542.

[Dierks and Rescorla 2008] Dierks, T. and Rescorla, E. (August 2008). The Transport Layer Security (TLS) Protocol Version 1.2. Technical report, RFC Editor. DOI: 10.17487/RFC5246.

[Eronen and Tschofenig 2005] Eronen, P. and Tschofenig, H. (December 2005). Pre-Shared Key Ciphersuites for Transport Layer Security (TLS). Technical report, RFC Editor. DOI: $10.17487 /$ RFC4279.

[Hassell 2002] Hassell, J. (2002). RADIUS: securing public access to private resources. " O’Reilly Media, Inc.".

[IEEE 2010] IEEE (2010). IEEE Standard for Local and metropolitan area networks - Port-Based Network Access Control. Technical report, IEEE. DOI 10.1109/ieeestd.2010.5409813, http://ieeexplore.ieee.org/servlet/opac?punumber=5409757.

[INEP-MEC 2019] INEP-MEC (2019). Censo Escolar. http: / / portal . inep . gov . br/censo-escolar. Accessed: 2020-09-09. 
[Presidencia da Republica, Brasil 1996] Presidencia da Republica, Brasil (1996). Lei $\mathrm{n}^{\mathrm{o}}$ 9.394, de 20 de dezembro de 1996: Estabelece as diretrizes e bases da educação nacional. http://www.planalto.gov.br/ccivil_03/leis/19394.htm.

[Rigney et al. 2000] Rigney, C., Willens, S., Rubens, A., and Simpson, W. (2000). Remote Authentication Dial In User Service (RADIUS). Technical report, RFC Editor. DOI: 10.17487/RFC2865.

[Rivoir Cabrera 2009] Rivoir Cabrera, A. (2009). Innovación para la inclusión digital. El Plan Ceibal en Uruguay. Mediaciones sociales, 1(4):299-328.

[Stanley et al. 2005] Stanley, D., Walker, J., and Aboba, B. (March 2005). Extensible Authentication Protocol (EAP) Method Requirements for Wireless LANs. Technical report, RFC Editor. DOI: 10.17487/RFC4017.

[Van der Walt 2011] Van der Walt, D. (2011). FreeRADIUS Beginner's Guide. Packt Publishing Ltd.

[Van Dijk 2006] Van Dijk, J. A. (2006). Digital divide research, achievements and shortcomings. Poetics, 34(4-5):221-235.

[Wierenga et al. 2015] Wierenga, K., Winter, S., and Wolniewicz, T. (September 2015). The eduroam Architecture for Network Roaming. Technical report, RFC Editor. DOI: 10.17487/RFC7593.

[Winter and McCauley 2015] Winter, S. and McCauley, M. (October 2015). Dynamic Peer Discovery for RADIUS/TLS and RADIUS/DTLS Based on the Network Access Identifier (NAI). Technical report, RFC Editor. DOI: 10.17487/RFC7585.

[Winter et al. 2012] Winter, S., McCauley, M., Venaas, S., and Wierenga, K. (May 2012). Transport Layer Security (TLS) Encryption for RADIUS. Technical report, RFC Editor. DOI: $10.17487 /$ RFC6414.

[Zhou et al. 2014] Zhou, H., Cam-Winget, N., Salowey, J., and Hanna, S. (May 2014). Tunnel Extensible Authentication Protocol (TEAP) Version 1. Technical report, RFC Editor. DOI: $10.17487 /$ RFC7170. 